\title{
Correspondence
}

\section{Adult ADHD as a dimensional disorder}

Moncrieff \& Timimi argue that there is no specific evidence to link adult attention-deficit hyperactivity disorder (ADHD) with childhood ADHD. ${ }^{1}$ They also question the increase in the use of stimulants for the condition and the role of the pharmaceutical industry in this. We can all lament the way in which the pharmaceutical industry has tried to increase the use of their products, yet the mere fact that they have done so does not invalidate their use.

The authors seem to ignore that most clinicians and academics see ADHD as a dimensional disorder. Just as with depression, the cut-off point for treatment is essentially arbitrary. This is the case in many psychiatric and other medical illnesses and conditions. We all recognise a patient when the illness is severe but it is less clear whether treatment is the appropriate course of action in less severe cases.

Majority opinion clearly suggests that the reason for the symptoms of ADHD is an increased density of dopamine transporter (DAT) complexes. ${ }^{2,3}$ With increasing age, there is a natural decline of these complexes, which causes a reduction of core symptoms. This leads to a change of prioritisation of core difficulties in adults, which does not represent a completely different set of symptoms as the authors suggest. The other argument the authors pursue is the high rate of comorbidity which they argue invalidates the diagnosis. However, untreated ADHD is likely to cause secondary difficulties such as conduct problems, personality disorder and substance misuse. Of course these difficulties cause some symptoms that are similar to the core symptoms of ADHD, but this hardly invalidates the primary diagnosis. More research is needed to find out whether adult treatment of ADHD mitigates the impact of acquired secondary problems. The current evidence would suggest that this is probably not the case. Therefore, the authors are certainly correct when they urge caution in the use of stimulants in adults if the main reason for the treatment would be to treat secondary diagnoses.

The authors argue that the wide variation of prevalence rates in difference studies is an argument against the validity of the concept of ADHD. However, such varieties are found in many dimensional syndromes. Depression and personality disorder are only two examples where this is the case. The American studies usually show higher prevalence rates because of their lower cut-off point for caseness of ADHD. In Europe, because the cut-off point is arbitrary and researches usually have it set higher, the prevalence figures appear different.

Moncrieff \& Timimi mention a follow-up study which, they claim, shows that any beneficial effects from stimulant use are not sustained at long-term follow-up. ${ }^{4}$ Careful analysis of this study would have shown that the reported lack of sustained benefit had to do with the relatively high drop-out rate in the intention-to-treat analysis. This is not surprising as most psychiatric studies over 3 years have high drop-out rates. However, the subgroup of children that stayed in this study and continued with their medication actually maintained the benefits throughout the 3-year period. I fully agree with the authors that the evidence in adults is rather less clear, although on current evidence the effect sizes of stimulant drugs are certainly among the highest in medicine.

At the end of the day, the decision to treat adult ADHD with stimulants is a clinical one that should take into account the severity of symptoms, potential side-effects, and the likelihood of reasonable improvement.

1 Moncrieff J, Timimi S. Critical analysis of the concept of adult attentiondeficit hyperactivity disorder. Psychiatrist 2011; 35: 334-8.

2 Del Campo N, Chamberlain SR, Sahakian BJ, Robbins TW. The roles of dopamine and noradrenaline in the pathophysiology and treatment of attention-deficit/hyperactivity disorder. Biol Psychiatry 2011; 69: e14557.

3 Lepping $P$, Huber $M$. Role of zinc in the pathogenesis of ADHD: implications for research and treatment. CNS Drugs 2010; 24: 721-8.

4 Jensen PS, Arnold LE, Swanson JM, Vitiello B, Abikoff HB, Greenhill LL, et al. 3-year follow-up of the NIMH MTA study. J Am Acad Child Adolesc Psychiatry 2007; 46: 989-1002.

Peter Lepping, Consultant Psychiatrist, Visiting Professor, Associate Medical Director, Betsi Cadwaladr University Health Board, North Wales, Glyndwr University, UK, email: peter.lepping@wales.nhs.uk

doi: $10.1192 / \mathrm{pb} .35 .12 .473$

\section{Are we missing the point in the debate on adult ADHD?}

There is no doubt attention-deficit hyperactivity disorder (ADHD) in adults is a relatively new concept and as the evidence base emerges it is a good idea to critically appraise it. It has its problems being a 'trait' condition where the traits are distributed across the spectrum in the population. This poses a challenge to clinicians on where to set the bar for illness. One can argue to what extent this process is influenced by societal values and expectations.

The dilemma of categorising a symptom present in continuum in the population into an illness and wellness dichotomy is not unique to ADHD or even to mental health. It resonates with issues faced in setting the bar for hypertension or hyperglycaemia.

Rather than getting into a critical analysis, Moncrieff \& Timimi $^{1}$ seem to have approached the subject in a one-sided way that tends towards not accepting the condition exits rather than objectively weighing up-to-date evidence. For example, they state: 'The evidence from randomised trials in adults and children therefore provides little basis for the sort of long-term drug treatment that is now being implemented for adults presenting with ADHD de novo, or for those with a continuation of a childhood presentation'. With regard to this statement, it is unclear who is recommending this.

The paper repeatedly quotes secondary research and uses qualitative remarks without systematically analysing data. Rather than looking into evidence base for current pharmacological treatment, the authors mention the National Institute for Health and Clinical Excellence guidance and focus on three randomised controlled trials quoted in that document. The recent Cochrane review on the matter found seven studies. ${ }^{2}$ 